\title{
On the coneighbor eigenvalues and coneighbor energy of a graph
}

\author{
Nechirvan Badal Ibrahim ${ }^{a}$ \\ ${ }^{a}$ Department of Mathematics, College of Science, University of Duhok, Kurdistan Region, Duhok, Iraq.
}

\begin{abstract}
In this paper, we define a new concept in theory of graph energy called coneighbor energy of a graph $G$, denoted by $\mathrm{CE}(\mathrm{G})$, as the sum of the absolute value of eigenvalues of its coneighbor matrix. We are going to derive some results about the coneighbor energy of graphs and coneighboracation graph that possess some coneighbor vertices.
\end{abstract}

Keywords: spectrum (of graph), energy (of graph), coneighbor spectrum, coneighbor energy.

2010 MSC: 05 C99

\section{Introduction}

Let $G$ be a simple undirected graph with $n$ vertices, and let $A(G)=\left[a_{i j}\right]$ be its adjacency matrix of a graph $G$. The eigenvalues $\lambda_{1}, \lambda_{2}, \cdots, \lambda_{n}$ of $A(G)$ are the (ordinary) eigenvalues of the graph $G$ studied in [3]. Since $A$ is a symmetric matrix with zero trace, these eigenvalues are real with sum equal to zero.

The concept of energy was first introduced in [4] The energy of a graph $E(G)$, is the sum of the absolute value of eigenvalues of its adjacency matrix. In [1] introduced the concept of common neighborhood energy of a graph $\mathrm{G}$ and obtained an upper bound for common neighborhood energy when $\mathrm{G}$ is strongly regular. Also showed that the common neighborhood energy of several classes of graphs is less than the common neighborhood energy of the complete graph. The energy of some composite graphs is studied in [7]. In [6] studied the Path Eigenvalues and Path Energy of Graphs. Information on the theory of graph and of graph energy can be found in $[2,5,9,10]$.

\section{Coneighbor Spectra of a Graph}

Definition 2.1. Let $u$ and $v$ be any two non-adjacent vertices and have the same neighbors in a graph $G$ are said to be coneighbor vertices in a graph $G$ that is $N_{G}(u)=N_{G}(v)$.

Definition 2.2. A coneighbor graph is a graph which contains the sets of coneighbor vertices.

Email address: nechirvan. badal@uod.ac (Nechirvan Badal Ibrahim)

doi:doi.org/10.31559/glm2020.9.2.2 
Definition 2.3. The coneighbor matrix $\operatorname{con}(\mathrm{G})$ or $\operatorname{con}=\left[\mathrm{c}_{\mathrm{ij}}\right]$ of a labeled graph $\mathrm{G}$ of order $\mathrm{p}$ and with vertex set $V(G), V(G)=\left\{v_{1}, v_{2}, \ldots, v_{p}\right\}$ is a $p \times p$ matrix in which $c_{i j}=1$ if $v_{i}$ and $v_{j}$ are coneighbor, and 0 if they are not. That is

$$
c_{i j}= \begin{cases}1 & \text { if } v_{i} \text { and } v_{j} \text { are coneighbor vertices } \\ 0 & \text { otherwise }\end{cases}
$$

The coneighbor matrix of a graph $\mathrm{G}$ is a symmetric 0,1 matrix having zero entries along the main diagonal, and in which the sum of the entries in any row or column is equal to the coneighbor vertices of the corresponding vertex. Because of this correspondence between graphs and matrices, any graph theoretic concept is reflected in the coneighbor matrix.

The eigenvalues $\mu_{1}, \mu_{2}, \cdots, \mu_{p}$ of a graph $G$ of order $p$ are defined to be the coneighbor eigenvalues of the coneighbor matrix associated with the graph $G$. That is, if $G$ has coneighbor matrix $\operatorname{con}(G)$, then the eigenvalues of $G$ are those $p$ (not necessarily distinct) numbers $\mu$ which satisfy the determinant equation $\operatorname{Det}\left(\operatorname{con}(G)-\mu I_{p}\right)=0$, viz each $\mu$ is a root of the polynomial equation $\left|\operatorname{con}(G)-\mu I_{p}\right|=0$. And can be ordered as $\mu_{1} \geqslant \mu_{2} \geqslant \cdots \geqslant \mu_{p}$.

The coneighbor spectrum $S p_{\text {con }}(G)$ of a graph $G$ is defined as the eigenvalues of its coneighbor matrix, that is, another matrix of two rows, the first row consists of the eigenvalues of the graph $G$ and the second row consists of the multiplicities of the corresponding eigenvalues. That is if the distinct eigenvalues of $G$ are $\mu_{1}, \mu_{2}, \cdots, \mu_{k}$ and their multiplicities are $m_{1}\left(\mu_{1}\right), m_{2}\left(\mu_{2}\right), \cdots, m_{k}\left(\mu_{k}\right)$, respectively, then we write

$$
\operatorname{Sp}_{\operatorname{con}}(G)=\left[\begin{array}{cccc}
\mu_{1} & \mu_{2} & \cdots & \mu_{k} \\
m_{1}\left(\mu_{1}\right) & m_{2}\left(\mu_{2}\right) & \cdots & m_{k}\left(\mu_{k}\right)
\end{array}\right]
$$

Or just as $\mu_{1}^{m_{1}}, \mu_{2}^{m_{2}}, \cdots, \mu_{k}^{m_{k}}$.

The coneighbor polynomial of the coneighbor matrix $\operatorname{con}(G)$ of a graph $G$ with $p$ vertices is called the coneighbor polynomial of $G$, denoted by $\phi_{\operatorname{con}}(G ; \mu)$ with the convention that the coefficient of the highest order term is positive:

$$
\phi_{\operatorname{con}}(G ; \mu)=\operatorname{Det}\left(\mu I_{p}-\operatorname{con}(G)\right)=(-1)^{p} \operatorname{Det}\left(\operatorname{con}(G)-\mu I_{p}\right) .
$$

Therefore, the coneighbor polynomial of a graph $\mathrm{G}$ of order $\mathrm{p}$ is a polynomial of degree $\mathrm{p}$ :

$$
\phi_{\text {con }}(G ; \mu)=a_{0} \mu^{p}+a_{1} \mu^{p-1}+\cdots+a_{p-1} \mu+a_{p} .
$$

Spectra of graphs can be obtained using the fact that the coefficients of the coneighbor polynomial are integers. It follows that the sum of $k$-th powers of eigenvalues are integers too. Since the coefficient of the highest power term $\mu_{p}$ of the coneighbor polynomial $\phi_{c o n}(G ; \mu)$ is equal to 1 , hence any eigenvalue of $\mathrm{G}$ which is rational must be an integer, and for any square matrix with real entries, the sum of its eigenvalues is equal to its trace.

Theorem 2.4. The coneighbor eigenvalues of a real symmetric coneighbor matrix are real.

Theorem 2.5. The sum of the coneighbor eigenvalues of any simple graph is zero.

Proof. The coneighbor matrix is a real symmetric matrix.

Theorem 2.6. Let $\mathrm{G}$ be the graph with coneighbor eigenvalues $\mu_{1}, \mu_{2}, \cdots, \mu_{\mathrm{p}}$, and let the coneighbor eigenvalues of $\mathrm{G}-v$ be $\lambda_{1}, \lambda_{2}, \cdots, \lambda_{\mathrm{p}-1}$. Then the coneighbor eigenvalues of $\mathrm{G}$ - vare interlaced with the coneighbor eigenvalues of $\mathrm{G}$ that is $\mu_{1} \geqslant \lambda_{1} \geqslant \mu_{2} \geqslant \lambda_{2} \geqslant \cdots \geqslant \lambda_{\mathrm{p}-1} \geqslant \mu_{\mathrm{p}}$.

Example 2.7. Consider the graphs $P_{3}, C_{4}$ and $K_{2,2}$ in below: 


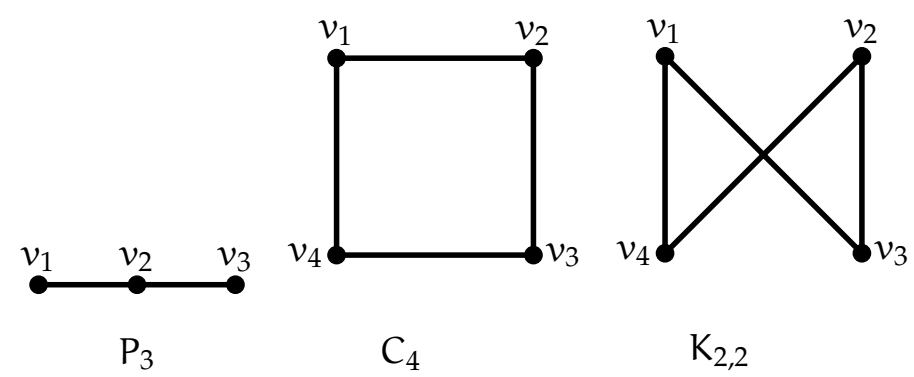

$\operatorname{con}(P-3)=\left[\begin{array}{lll}0 & 0 & 1 \\ 0 & 0 & 0 \\ 1 & 0 & 0\end{array}\right], \operatorname{con}\left(C_{4}\right)=\left[\begin{array}{llll}0 & 0 & 1 & 0 \\ 0 & 0 & 0 & 1 \\ 1 & 0 & 0 & 0 \\ 0 & 1 & 0 & 0\end{array}\right]$ and $\operatorname{con}\left(K_{2,2}\right)=\left[\begin{array}{llll}0 & 1 & 0 & 0 \\ 1 & 0 & 0 & 0 \\ 0 & 0 & 0 & 1 \\ 0 & 0 & 1 & 0\end{array}\right]$. And the coneighbor spectra of these graphs are $\operatorname{Spcon}\left(\mathrm{P}_{3}\right)=\left[\begin{array}{ll}1 & 0 \\ 2 & 1\end{array}\right], \operatorname{Spcon}\left(\mathrm{C}_{4}\right)=\left[\begin{array}{c}-1 \\ 4\end{array}\right]$ and $\operatorname{Spcon}\left(\mathrm{K}_{2,2}\right)=\left[\begin{array}{cc}1 & -1 \\ 2 & 2\end{array}\right]$.

Let $J_{p}$ the square matrix of order $p$, whose all non-diagonal elements are equal to one, and all diagonal elements are zero.

Proposition 2.8. If $\mathrm{G}$ is a disconnected graph with components $\mathrm{G}_{1}$ and $\mathrm{G}_{2}$, then the spectrum of $\mathrm{G}$ is the "union" of the eigenvalues of the components of $\mathrm{G}$.

Then, $\operatorname{con}(\mathrm{G})=\left[\begin{array}{cc}0 & \mathrm{~J}_{\mathrm{p}_{1}} \\ \mathrm{~J}_{\mathrm{p}_{2}} & 0\end{array}\right]$.

And

$$
\phi_{\text {con }}(G ; \mu)=\phi_{\text {con }}\left(G_{1} ; \mu\right) \phi_{\text {con }}\left(G_{2}, \mu\right) .
$$

Proposition 2.9. [10] The characteristic polynomial of complete graph is $\phi\left(K_{n}\right)=\left(\mu-(n-1)(\mu+1)^{n-1}\right)$.

And the energy of the complete graph $\mathrm{K}_{\mathrm{n}}$ is equal to $2(\mathrm{n}-1)$.

Proposition 2.10. 1. $\phi_{\text {con }}\left(K_{p_{1}, p_{2}} ; \mu\right)=\left(\mu-\left(p_{1}-1\right)\right)(\mu+1)^{p_{1}-1}\left(\mu-\left(p_{2}-1\right)(\mu+1)^{p_{2}-1}\right.$, if $p_{1}=p_{2}=p$, then $\phi_{\text {con }}\left(K_{p, p} ; \mu\right)=(\mu-(p-1))^{2}(\mu+1)^{2 p-2}$.

2. $\phi_{\text {con }}\left(S_{1, p} ; \mu\right)=\mu(\mu-(p-1))(\mu+1)^{p-1}$.

3. $\phi_{\operatorname{con}}\left(P_{p} ; \mu\right)= \begin{cases}\mu\left(\mu^{2}-1\right) & \text { if } p=3 \\ \mu^{p} & \text { if } p \geqslant 4 .\end{cases}$

4. $\phi_{\operatorname{con}}\left(C_{p} ; \mu\right)= \begin{cases}\left(\mu^{2}-1\right)^{2} & \text { if } p=4 \\ \mu^{p} & \text { if } p \geqslant 5 .\end{cases}$

5. $\phi_{\operatorname{con}}\left(K_{p} ; \mu\right)=\mu^{p}$.

Proof. 1. Let $K_{p_{1}, p_{2}}$ be the complete bipartite graph on order $p_{1}+p_{2}$, and let $V\left(K_{p_{1}, p_{2}}\right)=\left\{v_{1}, v_{2}, \cdots, v_{p_{1}}\right\}$ be the set of nonadjacent vertices of its one part and $U\left(K_{p_{1}, p_{2}}\right)=\left\{u_{1}, u_{2}, \cdots, u_{p_{2}}\right\}$ be the set of nonadjacent vertices of the other part. Then each vertex in $V\left(K_{p_{1}, p_{2}}\right)$ is adjacent to every vertices in $\mathrm{U}\left(\mathrm{K}_{\mathrm{p}_{1}, \mathrm{p}_{2}}\right)$.

Therefore, $\operatorname{con}\left(K_{p_{1}, p_{2}}\right)=\left[\begin{array}{ll}A\left(K_{p_{1}}\right) & 0_{p_{1} \times p_{2}} \\ 0_{p_{2} \times p_{1}} & A\left(K_{p_{2}}\right)\end{array}\right]$. We have: $\phi_{\operatorname{con}}\left(K_{p_{1}, p_{2}} ; \mu\right)=\operatorname{det}\left(\mu I-\operatorname{con}\left(K_{p_{1}, p_{2}}\right)\right)=$ $\left|\mu I-\left[\begin{array}{ll}A\left(K_{p_{1}}\right) & 0_{p_{1} \times p_{2}} \\ 0_{p_{2} \times p_{1}} & A\left(K_{p_{2}}\right)\end{array}\right]\right|$, where $0_{p_{1} \times p_{2}}$ and $0_{p_{2} \times p_{1}}$ be the $p_{1} \times p_{2}$ and $p_{2} \times p_{1}$ zero matrices, respectively. Which implies $\phi_{\text {con }}\left(K_{p_{1}, p_{2}} ; \mu\right)=\phi\left(K_{p_{1}} ; \mu\right) \phi\left(K_{p_{2}} ; \mu\right)$, by Proposition 2.9 , we get the result. And $\operatorname{Spcon}\left(K_{\mathrm{p}_{1}, \mathrm{p}_{2}}\right)=\left[\begin{array}{cccc}\mathrm{p}_{1}-1 & -1 & -1 & \mathrm{p}_{2}-1 \\ 1 & \mathrm{p}_{1}-1 & \mathrm{p}_{2}-1 & 1\end{array}\right]$. 
2. Let $S_{1, p}$ be the star graph of order $p+1$, and let $V\left(S_{1, p}\right)=\left\{v_{0}, v_{1}, v_{2}, \cdots, v_{p}\right\}$ be the set of vertices and $v_{0}$ is adjacent to every other vertices in $S_{1, p}$.

Therefore, $\operatorname{con}\left(S_{1, p}\right)=\left[\begin{array}{cc}0 & 0 \\ 0 & A\left(K_{p}\right)\end{array}\right]$, we have:

$$
\phi_{\operatorname{con}}\left(S_{1, p} ; \mu\right)=\operatorname{det}\left(\mu I-\operatorname{con}\left(S_{1, p}\right)\right)=\left|\mu I-\left[\begin{array}{cc}
0 & 0 \\
0 & A\left(K_{p}\right)
\end{array}\right]\right|
$$

Which implies $\phi_{\text {con }}\left(S_{1, p} ; \mu\right)=\mu \phi\left(K_{p} ; \mu\right)=\mu(\mu-(p-1))(\mu+1)^{p-1}$.

And $\operatorname{Spcon}\left(S_{1, p}\right)=\left[\begin{array}{ccc}p-1 & 0 & -1 \\ 1 & 1 & p-1\end{array}\right]$.

3. In path graph of order 3 there is one set of coneighbor vertices, by calculating we get the result, and if $p \geqslant 4$, we see that there is no two vertices are coneighbor.

4. In cycle graph of order 4 there is two sets of coneighbor vertices, by calculating we get the result, and if $p \geqslant 5$, we see that there is no two vertices are coneighbor.

5. In complete graph of order $\mathrm{p}$, we see that there is no two vertices are coneighbor.

\section{Coneighbor Energy of Graphs}

Definition 3.1. Let $\mu_{1}, \mu_{2}, \cdots, \mu_{k}$ be the eigenvalues of the coneighbor matrix of a graph $G$, then the coneighbor energy of a graph $G$ of order $p$ is defined as $C E=C E(G) \sum_{k=1}^{p}\left|\mu_{k}\right|$. It is clear that $C E(G) \geqslant 0$ and the equality hold if and only if $\mathrm{G}$ has no coneighbor vertices.

Proposition 3.2. If the graph $\mathrm{G}$ consists of (disconnected) components $\mathrm{G}_{1}, \mathrm{G}_{2}, \cdots, \mathrm{G}_{\mathrm{p}}$, then

$$
\mathrm{CE}(\mathrm{G})=\sum_{k=1}^{p} \mathrm{CE}\left(\mathrm{G}_{k}\right)
$$

Proposition 3.3. 1. $C E\left(K_{p_{1}, p_{2}}\right)=2 p_{1}+2 p_{2}-4$.

2. $C E\left(K_{p_{1}, p_{2}}-e\right)=2\left(p_{1}+p_{2}-6\right)$, for all $p_{1}, p_{2} \geqslant 3$.

3. $\operatorname{CE}\left(S_{1, p}\right)=2 p-2$.

4. $\mathrm{CE}\left(\mathrm{P}_{\mathrm{p}}\right)=\left\{\begin{array}{ll}2 & \text { if } \mathrm{p}=3 \\ 0 & \text { if } \mathrm{p} \geqslant 4\end{array}\right.$.

5. $\operatorname{CE}\left(\mathrm{C}_{\mathrm{p}}\right)=\left\{\begin{array}{ll}4 & \text { if } \mathrm{p}=4 \\ 0 & \text { if } \mathrm{p} \geqslant 5\end{array}\right.$.

6. $\mathrm{CE}\left(\mathrm{K}_{\mathrm{p}}\right)=0$.

7. $\operatorname{CE}\left(K_{p}-e\right)=2$.

Proof. 1. By Definition 3.1 and Proposition 2.10(1), we have

$$
\mathrm{CE}\left(\mathrm{K}_{\mathrm{p}_{1}, \mathrm{p}_{2}}\right)=\sum_{\mathrm{k}=1}^{\mathrm{p}_{1}+\mathrm{p}_{2}}\left|\mu_{\mathrm{k}}\right|=\mathrm{p}_{1}-1+\mathrm{p}_{1}-1+\mathrm{p}_{2}-1+\mathrm{p}_{2}-1=2 \mathrm{p}_{1}+2 \mathrm{p}_{2}-4
$$


2. If the vertices of $K_{p_{1}, p_{2}}$ are labeled so that all vertices $v_{1}, \cdots, v_{p_{1}}$ are adjacent to all vertices $v_{p_{1}+1}, \cdots, v_{p_{1}+p_{2}}$, and the deletion edge $e$ in is the edge between vertices $v_{p_{1}}$ and $v_{p_{1}+p_{2}}$, then

$$
\phi_{\operatorname{con}}\left(K_{p_{1}, p_{2}}-e ; \mu\right)=\operatorname{det}\left(\operatorname{con}\left(K_{p_{1}, p_{2}}-e\right)-\mu I\right)=\left|\left[\begin{array}{cc}
A\left(K_{p_{1}}-v\right) & 0 \\
0 & A\left(K_{p_{2}}-v\right)
\end{array}\right]-\mu I\right| .
$$

Which implies $C E\left(K_{p_{1}, p_{2}}-e\right)=E\left(K_{p_{1}-1}\right)+E\left(K_{p_{2}-1}\right)=2\left(p_{1}+p_{2}-6\right)$.

3. By Definition 3.1 and Proposition 2.10(2), we have:

$$
\operatorname{CE}\left(S_{1, p}, \mu\right)=\sum_{k=1}^{p+1}\left|\mu_{k}\right|=p-1+0+p-1=2 p-2 .
$$

4. Directly by Definition 3.1 and Proposition 2.10(3), we get the result.

5. Directly by Definition 3.1 and Proposition 2.10(4), we get the result.

6. Directly by Definition 3.1 and Proposition 2.10(5), we get the result.

7. If we remove one edge in $K_{p}$, we get one set of coneighbor vertices, then we get the result.

Theorem 3.4 (Coneighbor Energy). For any pair of coneighbor vertices $u$ and $v$ in a graph $\mathrm{G}$, then

$$
\mathrm{CE}(\mathrm{G})=\mathrm{CE}(\mathrm{G}-\mathrm{u})+2=\mathrm{CE}(\mathrm{G}-\mathrm{v})+2 .
$$

Proof. Label the vertices of $\mathrm{G}$ as $u=v_{1}, v=v_{2}, v_{3}, \cdots, v_{\mathrm{p}}$. Let $\operatorname{con}(\mathrm{G})$ is a square $\mathrm{p} \times \mathrm{p}$ matrix, $\operatorname{con}(\mathrm{G}-\mathrm{u})$ and $\operatorname{con}(G-v)$, be the coneighbor matrices of $G, G-u$ and $G-v$, respectively. Applying elementary operations by removing one of pairs of coneighbor vertex in any rows or in any columns of $\operatorname{con}(\mathrm{G})$, we get $\operatorname{con}(G-u)$ or $\operatorname{con}(G-v)$ are the square $(p-1) \times(p-1)$ matrices, this makes deleted one root and one column contains a coneighbor vertex with $u$ or with $v$ in $\operatorname{con}(G)$ this implies that we removed two eigenvalues in $\operatorname{con}(G)$. Thus, $C E(G)=C E(G-u)+2=C E(G-v)+2$.

Corollary 3.5. For each pair of coneighbor vertices $u$ and $v$ in a graph $G$, then $C E(G-u)=C E(G-v)$.

Proof. Follows from Theorem 3.4.

Theorem 3.6. If $\mathrm{u}_{1}, \mathrm{u}_{2}, \cdots, \mathrm{u}_{\mathrm{p}}$ are non-coneighbor vertices of a graph $\mathrm{G}$, then $\mathrm{CE}(\mathrm{G})=0$.

Corollary 3.7. If e is an edge between the neighborhood vertices in a coneighbor graph $\mathrm{G}$, then $\mathrm{CE}(\mathrm{G}-\mathrm{e})>\mathrm{CE}(\mathrm{G})$.

For example,

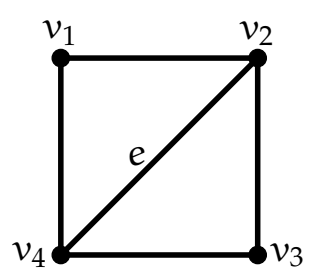

G

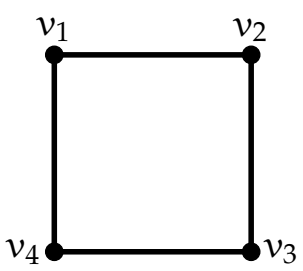

$\mathrm{G}-\mathrm{e}=\mathrm{C}_{4}$

Hence, $\mathrm{E}(\mathrm{G})=2<\mathrm{CE}((\mathrm{G}-2)=\mathrm{C} 4)=4$. 


\section{Coneighbor Energy of Coneighboracation Graph}

In this section, we obtained the coneighbor energy of coneighboracation graph between some special graphs. In [8] defined the Coneighboracation graph between two graphs and studied some properties and nullity of this graph.

Definition 4.1. Let $u_{1} \in G_{1}$ and $u_{2} \in G_{2}$, then, the coneighboracation graph $G_{1} @ G_{2}$ of $G_{1}$ and $G_{2}$ is the graph whose vertex set is $V\left(G_{1}\right) \cup V\left(G_{2}\right)$ and

$$
\mathrm{E}\left(\mathrm{G}_{1} \odot \mathrm{G}_{2}\right)=\mathrm{E}\left(\mathrm{G}_{1}\right) \cup \mathrm{E}\left(\mathrm{G}_{2}\right) \cup\left\{x \mathrm{u}_{2} \cup y u_{1}, x \in \mathrm{N}_{\mathrm{G}_{1}}\left(\mathrm{u}_{1}\right), y \in \mathrm{N}_{\mathrm{G}_{2}}(\mathrm{u} 2)\right\} .
$$

Theorem 4.2. The coneighbor energy of coneighboracation graph $\mathrm{G}_{1} @ \mathrm{G}_{2}$ is given by

$$
\mathrm{CE}\left(\mathrm{G}_{1} \odot \mathrm{G}_{2}\right)=2+\mathrm{CE}\left(\left(\mathrm{G}_{1}-\mathrm{u}_{1}\right) \bullet \mathrm{G}_{2}\right) .
$$

Proof. Let $\mathrm{u}_{1} \in \mathrm{G}_{1}$ and $\mathrm{u}_{2} \in \mathrm{G}_{2}$, in the coneighboracation graph $\mathrm{G}_{1} @ \mathrm{G}_{2}$ and thus $\mathrm{u}_{1}$ is a coneighbor vertex with $u_{2}$, then applying Theorem 3.4 by removing $u_{1}$ in $G_{1} \odot G_{2}$ we get:

$$
\mathrm{CE}\left(\mathrm{G}_{1} \odot \mathrm{G}_{2}\right)=\mathrm{CE}\left(\left(\mathrm{G}_{1} \odot \mathrm{G}_{2}\right)-\mathrm{u}_{1}\right)+2=2+\mathrm{CE}\left(\left(\mathrm{G}_{1}-\mathrm{u}_{1}\right) \bullet \mathrm{G}_{2}\right) .
$$

The vertex identification graph $G_{1} \bullet G_{2}$ is obtained from $G_{1}$ and $G_{2}$ by identifying the vertex $u \in G_{1}$ with $v \in \mathrm{G}_{2}$.

In the following proposition, we illustrate some cases.

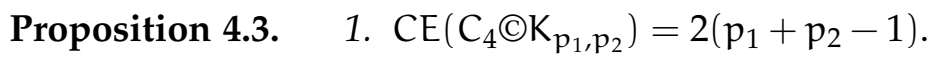

2. $\mathrm{CE}\left(\mathrm{C}_{4} @ \mathrm{~S}_{1, \mathrm{p}}\right)=2(\mathrm{p}+1)$.

3. $\operatorname{CE}\left(\mathrm{C}_{4} @ \mathrm{~K}_{\mathrm{p}}\right)=4$.

4. $\mathrm{CE}\left(\mathrm{C}_{4} \odot \mathrm{C}_{\mathrm{p}}\right)=\left\{\begin{array}{ll}6 & \text { if } \mathrm{p}=4 \\ 4 & \text { if } \mathrm{p} \geqslant 5\end{array}\right.$.

5. $\mathrm{CE}\left(\mathrm{C}_{4} \odot \mathrm{P}_{\mathrm{p}}\right)=\left\{\begin{array}{ll}6 & \text { if } \mathrm{p}=3 \text { and the identification at the vertex of degree two } \\ 4 & \text { otherwise }\end{array}\right.$.

Proof. For all cases applying Theorem 3.4 and using Proposition 3.3 we get the results.

Proposition 4.4. The coneighbor energy of coneighboracation graph $\mathrm{K}_{\mathrm{n}_{1}, \mathrm{n}_{2}} \odot \mathrm{K}_{\mathrm{m}_{1}, \mathrm{~m}_{2}}$ is given by

$$
\mathrm{CE}\left(\mathrm{K}_{\mathrm{n}_{1}, \mathrm{n}_{2}} \odot \mathrm{K}_{\mathrm{m}_{1}, \mathrm{~m}_{2}}\right)=2\left(\mathrm{n}_{1}+\mathrm{n}_{2}+\mathrm{m}_{1}+\mathrm{m}_{2}-5\right) .
$$

Proof. Applying Theorem 3.4, we have:

$\mathrm{CE}\left(\mathrm{K}_{\mathrm{n}_{1}, \mathrm{n}_{2}} \odot \mathrm{K}_{\mathrm{m}_{1}, \mathrm{~m}_{2}}\right)=\mathrm{CE}\left(\left(\mathrm{K}_{\mathrm{n}_{1}, \mathrm{n}_{2}}-\mathrm{u}_{1}\right) \odot \mathrm{K}_{\mathrm{m}_{1}, \mathrm{~m}_{2}}\right)=2+\mathrm{CE}\left(\mathrm{K}_{\mathrm{n}_{1}, \mathrm{n}_{2}} \bullet \mathrm{K}_{\mathrm{m}_{1}, \mathrm{~m}_{2}}\right)$, and applying Theorem 3.4, $\left(n_{1}-1\right)$ and $\left(n_{2}-2\right)$ times, we have:

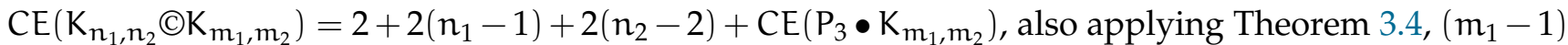
and $\left(m_{2}-2\right)$ times, we have: $C E\left(K_{n_{1}, n_{2}} \odot K_{m_{1}, m_{2}}\right)=2+2\left(n_{1}-1\right)+2\left(n_{2}-2\right)+2\left(m_{1}-1\right)+2\left(m_{2}-2\right)+$ $\mathrm{CE}\left(\mathrm{P}_{5}\right)$, And by Proposition 3.3(4), we get: $\mathrm{CE}\left(\mathrm{K}_{\mathrm{n}_{1}, \mathrm{n}_{2}} \mathrm{CK} \mathrm{K}_{\mathrm{m}_{1}, \mathrm{~m}_{2}}\right)=2\left(\mathrm{n}_{1}+\mathrm{n}_{2}+\mathrm{m}_{1}+\mathrm{m}_{2}-5\right)$. 


\section{References}

[1] A. Alwardi, N. D. Soner, I. Gutman, On the common-neighborhood energy of a graph, Bull. Acad. Serbe Sci. Arts (Cl. Math. Natur.), 143 (2011) 49-59. 1

[2] A. E. Brouwer, W. E. Haemers, Spectra of Graphs, Springer, New York, 2010. 1

[3] D. Cvetkovic, M. Doob and H. Sachs, Spectra of Graphs, Academic Press, New York, 1980. 1

[4] I. Gutman, The energy of a graph, Ber.Math. Stat. Sekt. Forschungsz. Graz 103 (1978) 1-22. 1

[5] M. L. Kragujevac, On the Laplacian energy of a graph, Czechoslovak Math.Jour. 56(131) (2006), 1207-1213. 1

[6] M. M. Shikare, P. P. Malavadkar, Sh. C. Patekar and I. Gutman, On Path Eigenvalues and Path Energy of Graphs, MATCH Commun. Math. Comput. Chem. 79 (2018) 387-398. 1

[7] ] N. B. Ibrahim, On the Energy of some Composite Graphs, Journal of University of Zakho, 3(A), No.1, (2015) 132-139. https:/ / doi.org/10.25271/2015.3.1.286 1

[8] N. B. Ibrahim, On the Nullity of Some Sequential Element Identified, Element Introduced Graphs. M.Sc. Thesis, University of Zakho, Iraq, 2013. 4

[9] S. C. Patekar and M. M. Shikare, On the path matrices of graphs and their properties, Adv. Appl. Discr. Math. 17 (2016) 169-184. https://doi.org/10.17654/dm017020169 1

[10] X. Li, Y. Shi, I. Gutman, Graph Energy, Springer, Berlin, 2012. 1, 2.9 